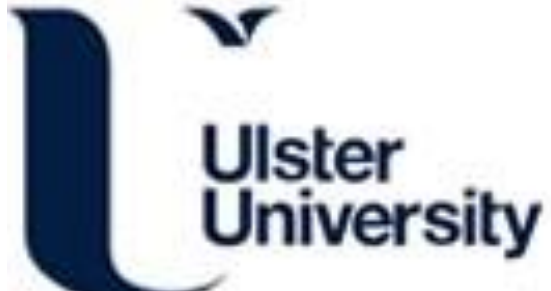

Moving with Beats and Loops: The Structure of Auditory Events and Sensorimotor Timing

Rodger, M. W. M., \& Craig, C. M. (2014). Moving with Beats and Loops: The Structure of Auditory Events and Sensorimotor Timing. In M. Aramaki, O. Derrien, R. Kronland-Martinet, \& S. Ystad (Eds.), Sound, Music, and Motion -10th International Symposium, CMMR 2013 (Vol. 8905, pp. 204-217). (Lecture Notes in Computer Science). Springer. https://doi.org/10.1007/978-3-319-12976-1_13

Link to publication record in Ulster University Research Portal

Published in:

Sound, Music, and Motion -10th International Symposium, CMMR 2013

Publication Status:

Published (in print/issue): 01/12/2014

DOI:

10.1007/978-3-319-12976-1_13

\section{Document Version}

Author Accepted version

\section{General rights}

Copyright for the publications made accessible via Ulster University's Research Portal is retained by the author(s) and / or other copyright owners and it is a condition of accessing these publications that users recognise and abide by the legal requirements associated with these rights.

\section{Take down policy}

The Research Portal is Ulster University's institutional repository that provides access to Ulster's research outputs. Every effort has been made to ensure that content in the Research Portal does not infringe any person's rights, or applicable UK laws. If you discover content in the Research Portal that you believe breaches copyright or violates any law, please contact pure-support@ulster.ac.uk. 


\section{QUEEN'S
UNIVERSITY
BELFAST}

\section{Moving with Beats and Loops: The Structure of Auditory Events and Sensorimotor Timing}

Rodger, M. W. M., \& Craig, C. M. (2014). Moving with Beats and Loops: The Structure of Auditory Events and Sensorimotor Timing. In M. Aramaki, O. Derrien, R. Kronland-Martinet, \& S. Ystad (Eds.), Sound, Music, and Motion -10th International Symposium, CMMR 2013 (Vol. 8905, pp. 204-217). (Lecture Notes in Computer Science; Vol. 8905). Springer. https://doi.org/10.1007/978-3-319-12976-1_13

Published in:

Sound, Music, and Motion -10th International Symposium, CMMR 2013

\section{Document Version:}

Peer reviewed version

Queen's University Belfast - Research Portal:

Link to publication record in Queen's University Belfast Research Portal

\section{Publisher rights}

(C) 2014 Springer International Publishing AG

he final publication is available at Springer via http://link.springer.com/chapter/10.1007\%2F978-3-319-12976-1_13

\section{General rights}

Copyright for the publications made accessible via the Queen's University Belfast Research Portal is retained by the author(s) and / or other copyright owners and it is a condition of accessing these publications that users recognise and abide by the legal requirements associated with these rights.

Take down policy

The Research Portal is Queen's institutional repository that provides access to Queen's research output. Every effort has been made to ensure that content in the Research Portal does not infringe any person's rights, or applicable UK laws. If you discover content in the Research Portal that you believe breaches copyright or violates any law, please contact openaccess@qub.ac.uk. 


\title{
Moving with Beats and Loops: the Structure of Auditory Events and Sensorimotor Timing
}

\author{
Matthew W. M. Rodger ${ }^{1}$ and Cathy M. Craig ${ }^{1}$, \\ ${ }^{1}$ School of Psychology, Queen’s University Belfast, Belfast, UK \\ m.rodger@qub.ac.uk \\ cathy.craig@qub.ac.uk
}

\begin{abstract}
.
Traditionally, audio-motor timing processes have been understood as motor output from an internal clock, the speed of which is set by heard sound pulses. In contrast, this paper proposes a more ecologically-grounded approach, arguing that audio-motor processes are better characterized as performed actions on the perceived structure of auditory events. This position is explored in the context of auditory sensorimotor synchronization and continuation timing. Empirical research shows that the structure of sounds as auditory events can lead to marked differences in movement timing performance. The nature of these effects is discussed in the context of perceived action-relevance of auditory event structure. It is proposed that different forms of sound invite or support different patterns of sensorimotor timing. Hence, the temporal information in looped auditory signals is more than just the interval durations between onsets: all metronomes are not created equal. The potential implications for auditory guides in motor performance enhancement are also described.
\end{abstract}

Keywords: sound structure, perceptual events, movement timing, sensorimotor synchronization

\section{Introduction}

As humans, we are uniquely able to time our movements to patterns of sounds with a high degree of accuracy, as exemplified by dancers, marching bands, and ensemble musicians. In order to understand this complex ability better, it is necessary to ask how our sensorimotor systems pick up information about time through auditory sensation, and use this information to control ongoing movements. It is the position of the present paper that this question is best approached by considering sounds as perceptual events, the different structures of which support or invite different forms of movement timing. This approach is here explained, with examples given of empirical studies that investigate the relationships between auditory event structure and timing of actions.

Historically, the predominant paradigm for studying the timing of movements to sounds (and other sensory timing cues) has been to ask experimental participants to tap in time with a metronomic stimulus, and/or continue tapping in time after the 
stimulus presentation has ceased [31]. There is a seemingly reductionist appeal of this approach: a discrete, intermittent sound constitutes a pure, uncluttered temporal interval as a stimulus; while a finger tap is a small enough response to minimize the disruption to timing from a noisy, dumb motor system. Hence, extraneous variables in the sensorimotor timing process can be minimized or removed, giving a 'true' picture of the underlying mechanisms involved. Factors in the input stimulus that are often varied in this research paradigm include interval duration between metronome tones, slight offsets from isochrony of consecutive tones, or presentation of timing cues to different sensory modalities [29, 30, 48]. In turn, mathematical models of timing errors in motor output are generated to explain the supposed underlying processes in participants' synchronization performance. A common assumption seems to be that there is a clock-like process somewhere in the mind [45, 47], i.e. a regular intermittent pulse-like signal that transmits timing information to wherever it is needed in the brain. It is further assumed that by stripping back the movement timing task to its simplest form, the characteristics of this mental clock can be discerned by peering through the tea-leaves of messy perceptual and motor systems.

Although somewhat unfairly caricatured, the above description portrays a prevalent theoretical stance towards researching movement timing in relation to sounds. In contrast, we believe that this approach fails to capture important characteristics of how people move with sounds in the real-world. For example, movements of larger scales than finger-tapping can successfully be performed in time with auditory events, e.g. a percussionist striking a timpani drum can require movement of her full arm. Furthermore, sounds in the world rarely occur as metronomic pure tone islands in a sea of silence, but rather in a continuous unfolding stream, the structure of which varies in pitch, intensity, timbre and other auditory properties. With this in mind, it is important to think of sound-based timing cues not as extension-less points on an artificial timeline, but as events; an event being something that "occupies a restricted portion of four-dimensional space-time" [10]. Thus, to fully understand the capacity to move in time with sounds, it is necessary to consider a broader range of perceptual and motion factors in one's experimental investigations. At a more theoretically fundamental level, the established picture of sensorimotor synchronization as sensory input $\rightarrow$ mental clock processing $\rightarrow$ motor output belies the embodied, dynamic nature with which we interact with our perceptual environments $[6,12,18]$.

These concerns may be remedied by adopting a more ecological stance to the questions of how we are able to time our actions to sounds. The ecological approach in psychology considers agents (people/organisms) as active perceivers of their surrounding environments [16]. Moreover, environments give a structure to the energy (e.g. light, air vibrations) that excite sensory organs (e.g. retina, cochlea), such that patterns of sensation are specific to the geometric properties of objects, surfaces and events that cause them [42]. On the whole, these world-specifying patterns are not clear from a static perspective, but reveal themselves through ongoing movement, such as how the visual field moves as we move our eyes. This means that there is sensory information available for active perceivers that directly informs about events in the world [38]. In the case of sounds, patterns of vibration in the air are specific to the events (scrapes, collisions, drops...) that give rise to them [14]. Furthermore, auditory events are not isolated atoms of sensation in time, but rather they unfold temporally, and the form of this unfolding is essential for perceiving the nature of sound-causing physical interactions [4]. Finally, the ecological approach in 
psychology puts a firm emphasis on action, asserting that the job of perception is to help us move successfully through the world [16]. In this picture, the brain is not an indifferent processor of detached sense data, but rather it is a tuning fork for action, selecting appropriate responses to the changing environmental scene [5]. Hence, perceptual information is constrained and defined by how it invites or dictates particular actions [49].

Considering this theoretical framework - one that is more ecological / embodied in perspective - the important questions for our current purposes should be: how do the structures of auditory events support or invite time-sensitive actions, and conversely, how do people control timed movements for different event-specifying sounds? By adopting this approach, it is still possible to scientifically investigate the manner in which auditory factors can give rise to different movement timing behaviors in a controlled, rigorous manner. For example, most of the research presented here involves a paradigm in which participants are asked to make a number of repetitive upper limb movements at a rate that matches the tempo specified by different looping sounds. An important step in this process is considering the structure of sound in a perceptual event, that is, how do auditory parameters (e.g. pitch, intensity) change over time, and how might differences in these structure (e.g. onset attack, slope) be meaningfully related to action. By generating synthetic sounds that possess clear differences in auditory structure, and looking at subsequent differences in performance when participants have to perform simple actions in time with such sounds, one can carefully study the relationship between auditory events and sensorimotor timing. The proposed advantage of framing such an investigation in this way is that it allows experimental conclusions to be drawn that will more likely provide insight into how sounds guide actions in real-world scenarios.

\section{Extrinsic and Intrinsic Information for Action}

If we are to consider the timing of action as guided by the available information specifying temporal intervals, it is important to consider the nature of this information and where it comes from. A useful framework for addressing this question can be found in the outline of David Lee's General Tau Theory [20-22]. According to this theory, the control of any action (big or small) can be thought of as the closure of a motion gap between the limb's current state and its goal state. For example, catching a ball involves closing the motion gap between one's hand and the ball's trajectory. Successful actions involve closing this motion gap within a suitable temporal gap, i.e., getting to the right place at the right time. For example, playing a drum in time involves closing the motion gap between the end of the stick/beater and the surface of the drum within the temporal gap that closes between consecutive beats. More complex movements may involve the closure of multiple motion gaps concurrently, e.g. marching in time with others involves closing the motion gap between foot and ground at the same instant that those around you do the same. The sensorimotor control of this process, therefore, involves coupling the closure of motion gaps to appropriate dynamic information that will lead to the successful spatial-temporal execution of an action. 
An important question at this stage is where within the agent-environment system the information is found with which to guide the closure of a given motion gap. According to the theory, a distinction can be made between extrinsic or intrinsic information for movement guidance. Extrinsic information entails a relevant, eventspecifying sensory gap to which the unfolding motion gap may be coupled. For example, in the case of catching a ball, the sensory gap exists visually between the ball and the hand. By coupling these together, such that both gaps close to zero at the same time, a successful interceptive action will be achieved. Hence, extrinsic guidance of movement implies that the information is available in the dynamics of events in our environment. When there is not information available in the environment to guide closure of the relevant motion gap, as in the case of self-paced actions, intrinsic information must be generated within the nervous system. For example, a golfer making a putt has no external timing information to guide her swing, and so must use an unfolding internally-generated neural gap to control her action [7]. Thus, although intrinsic and extrinsic guidance of action involve different processes, both can be characterized as the coupling of movement to information about a closing spatial-temporal gap [20].

General Tau Theory goes further to say that the nature of the informational variable used for both extrinsic and intrinsic guidance of action is the time-to-closure of a gap (motion, sensory, neural) at its current rate of closure. This variable, tau, can be directly sensed as the ratio between the current magnitude of a gap and its current rate of closure. In the case of extrinsic guidance, the tau of the motion gap can be coupled to the tau of a sensory gap, such as the visual angle on the retina relative to its rate of change [20]. In the case of intrinsic guidance, a motion tau is coupled to the neural power gap, relative to its rate of change [21]. Without going into too much detail, it can be noted that there is a great deal of support for this variable in the control of actions in which there is external dynamic sensory information, such as, long-jumpers taking run-ups [23] or drivers controlling the timing of braking [19], as well as for actions that require internal guidance, such as, infants sucking [8], singers initiating vocal pitch glides [37], and musicians' expressive performance gestures [35] $]^{1}$. The important point for the present purposes is that General Tau Theory carves sensorimotor control of action into two distinct forms (extrinsic and intrinsic), depending on the availability of perceptual information for online guidance of movement, while also offering a candidate informational 'currency' that may be used in both cases.

\subsection{Discrete and Continuous Sounds}

Following the preceding discussion, it is possible to unpack the distinction between intrinsic and extrinsic information for guiding action in the context of synchronizing movement with sound. As mentioned in the introduction, a common sound type for assessing sensorimotor synchronization is a metronome, the general form of which is a short tone, followed by a period of silence, repeated at a set inter-onset interval. Although the presence of intermittent tones may be considered an extrinsic source of

${ }^{1}$ For a more comprehensive list of empirical studies relating to General Tau Theory, please visit http://www.pmarc.ed.ac.uk (Last accessed: 24/06/2013). 
timing information, there is no information about the unfolding temporal gap between tone onsets with which to couple individual movements (e.g. taps). Thus, according to present theory, each metronome tone is a discrete event, and the information for timing movements between individual tones must be neurally generated [9]. An alternative way to present cues for time intervals is to have a sound that changes along some auditory parameter (e.g. pitch or intensity) continuously throughout the duration of each interval, looping back to the initial parameter at the end of the duration. Fig. 1a illustrates the distinction between these two interval-specifying sound types. For each discrete interval, because there is no sensory information between tones, an intrinsic neural guide must fill in the temporal gap onto which the motion gap is coupled [9]. For each continuous interval, there is sensory information present in the changing auditory parameter about the unfolding temporal gap. Hence, according to the present theory, these different sound structures would likely recruit different movement timing processes.

a)

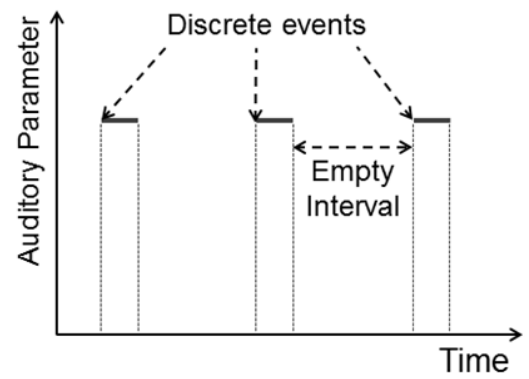

b)

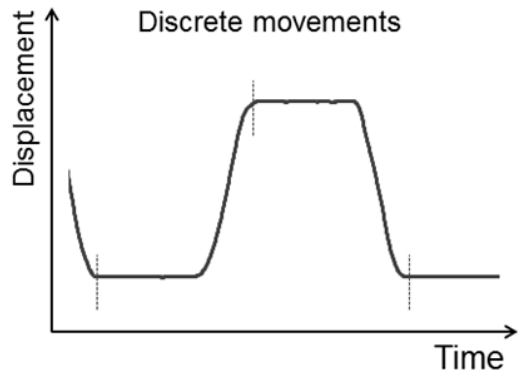

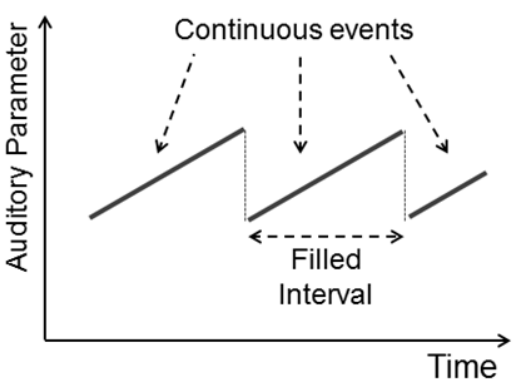

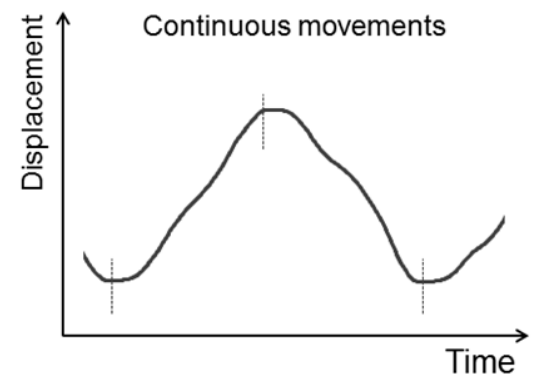

Fig 1. Representation of sound and movement structures across time. A): Discrete and continuous sound events represented as changes in auditory parameters against time. Discrete sound events (e.g. metronome) are represented as instantaneous changes to a set auditory parameter (tone, intensity, etc.) followed quickly by instantaneous change to zero, separated in time by empty (silent intervals). Continuous sound events (e.g. pitch ramp loops) are represented as constant change in the value of an auditory parameter during each interval, in this case with an instantaneous change to the initial value at interval boundaries. B): Movement represented as displacement of a point on a limb over time. In these examples, movement is of an index finger between two lateral targets. Data is taken from a participant synchronizing with discrete and continuous sound events shown in A with $2.5 \mathrm{~s}$ intervals. Discrete movements involve waiting in target zones for the majority of the interval with quick movements between targets to intercept subsequent beats, while continuous movements involve gradual change in 
displacement throughout each of the interval durations. Dashed vertical lines indicate synchronization points with sounds.

By plotting auditory parameters as a function of time, one can characterize the structure of each sound as differently shaped perceptual events, and in turn, consider the structure of different movements that may be invited by a particular sound structure $^{2}$. As shown in Fig. 1b, discrete sounds may be better suited to discrete movements (quick changes in displacement followed by recuperation or waiting), whereas continuous sounds may more likely invite smoother continual change in displacement over time. It is worth noting that tapping can be thought of as a discrete movement with a sharp change in displacement to the target surface followed by recuperation and pausing between subsequent actions. Hence, asking participants to tap to sound may bias the form of the observed movement trajectory. In cases where the form of movement to be made by a participant is less strictly specified by the task, as is the case in moving between lateral targets, different movement strategies can be found [2].

Rodger and Craig [34] reported a study which investigated the idea that different sound structures (discrete vs. continuous) can differentially affect timing and movement trajectory in a sensorimotor synchronization task. Participants $(n=23)$ had to repeatedly move their index finger reciprocally between two separate targets in synchrony with either discrete tones or looped continuous pitch ramp sounds. Different interval durations (temporal gaps) were used $(1 \mathrm{~s}, 2.5 \mathrm{~s}, 4 \mathrm{~s})$ and movement distances between targets (motion gaps) were also varied $(20 \mathrm{~cm}$ and $70 \mathrm{~cm})$. Results showed differences in sensorimotor synchronization between sounds types: timing errors were greater for continuous sounds (arriving in target too early), but variability of timing errors was lower in this condition, when compared with discrete sounds. Moreover, movements between targets were more harmonic in form (the relationship between velocity and displacement was closer to sinusoidal) for continuous sounds than discrete. Thus, the difference in structure of sound led to marked differences in sensorimotor synchronization performance and shape of movement. These effects were most pronounced for longer interval durations, suggesting that auditory-driven differences in intrinsic vs. extrinsic movement timing processes have a greater effect when temporal gaps are larger. It can be noted that these findings from Rodger and Craig [34] are supported by results from an experiment by Varlet et al. [43], which found similar changes in movement timing and trajectory when synchronizing wrist rotation movements with discrete (metronomic) and continuous (pitch oscillating) sounds.

In terms of relating the movement timing behavior to the sounds as auditory events, the differences in the shape of movement between targets may be understood as differences in the type of action invited by different auditory structures, as depicted in Fig. 1. However, it is still necessary to explain differences in timing caused by sound structure, that is, synchronization error and variability. This may be addressed by considering the information specifying perceptual event boundaries. Phillips et al. [28] provide evidence that sound intensity onsets mark the edges of new perceptual events. Discrete sounds have clear intensity (and technically, pitch) onsets at the start of each interval, marking a clear boundary for the perceptual event. For the

${ }^{2}$ More complex sound events may be thought of as multiple dimensions of auditory parameters changing over time relative to each other. 
continuous pitch ramps, the intensity is constant, while the instantaneous pitch drop at the end of each interval may form a more ambiguous event boundary than that created by discrete tones. This could explain why synchronization errors were smaller for discrete sounds ${ }^{3}$. The reduced variability in synchronization errors for continuous sounds may have to do with the more oscillatory nature of the ongoing perceptual events. That is, the overarching temporal form of the ongoing sound is consistently maintained in movement timing, without individual actions necessarily lining up neatly with individual event boundaries. In other terminology, there is a consistent but non-zero phase relationship between the movement and sound in this condition. The important point is that by considering the time-related structure of these different auditory events, it is possible to offer an account of observed effects on the temporal aspects of corresponding actions.

\subsection{Perceptually-Driven Changes in Motor Timing Behavior}

The present approach to understanding movement timing and the structure of sound events can also speak to a distinction often made in the motor control literature: that of event-based and emergent timing $[11,24]$. Tasks in which participants have to, for example, draw circles continuously in time with a metronome - as compared to tapping - result in markedly different patterns of timing error and movement trajectory [13, 39]. Typically, temporal errors are greater and less self-correcting for emergent timing (e.g. circle drawing) than for event-based timing (e.g. tapping). There is some debate as to whether this reflects different motor timing processes, or is the manifestation of two extremes of the same pulse-based motor control system [11, 33]. What is often not considered is the manner in which the action matches the available perceptual information specifying time, i.e. the metronome. If the timing information was presented as continual sound, would this lead to improvements in synchronization in emergent-timing tasks, relative to discrete sounds for timing? This question draws attention to the limitations of solely focusing on motor outputs to understand movement timing in response to sound cues.

More generally, the current position would encourage that one asks what is the information available to control movement, and how does this information invite a particular form of action over another. By characterizing sensorimotor synchronization problems in this way, resulting answers should be more commensurate with the way behavior is guided in real-world, non-laboratory situations.

\footnotetext{
${ }^{3}$ Another possible explanation is that the increasing tone and intensity ramps were perceived as looming sounds (objects approaching the listener), which have been shown to bias action responses to be earlier [26]. The explanation given for this effect is that looming sounds are likely to specify a prospective collision, and so we are more likely to respond early to avoid this. Although this account signifies the relevance of the ecological meaning of sounds in influencing actions, the fact that the pitch ramps were looped would likely mean that any looming effect would likely be attenuated by repetition.
} 


\section{Structure of Auditory Events and Re-enacted Duration}

In addition to cases where movement is synchronized with sound, there may also be scenarios in which sound is used to set-up a movement timing pattern for subsequent action. As an anecdotal example, imagine a footballer singing a familiar song to herself as a way to get the timing right in the run-up for a penalty kick. In such a case, the temporal structure of the sound resonates in the timing of the following action. Scientifically, the effects of timing cues on subsequent motor actions is often studied in the context of a continuation paradigm in which participants are presented with a metronomic stimulus and then asked to maintain the stimulus tempo after the sound has stopped [31]. As with the previous discussion, a relevant question for the present position is how the structure of a given sound within a timesetting interval may affect the subsequent continuation motor timing. That is, how does the sound resonate in the action after it has sounded? There is evidence that the structural content of an interval does influence perceived and enacted timing, and the current account offers a potential explanation for why this may be so.

It has been established that the content of two different sound events can affect their perceived durations, even if the physical durations are identical. Looming sounds are perceived as longer than receding sounds [17]; while emotionally negative sounds last longer perceptually than neutral ones [27]. A particularly well-studied instance of this general phenomenon is the observation that filled intervals have greater perceived duration than empty ones [3, 41,46]. For example, a continuous tone in an interval marked by silent gaps will be perceived as longer than a silent interval marked by short tones [46]. Also, discrete tone intervals are perceived as longer depending on the number of sub-interval tones they contain [3]. Thus, perceptual time is not a faithful recreation in the mind of the 'physical passing' (the regular passage of a clockmeasured fourth dimension), but rather it is modulated by the sensory information contained within an event. A putative explanation of this phenomenon is that the magnitude of energy/work specified by a sound event dilates the perception of that events duration. Hence, intervals that contain sustained energy, changing parameters, or sub-events will be perceived as lasting longer than more empty/static intervals, as these factors imply work of some kind on the part of the sound generator. This phenomenon carries into motor timing behavior. Repp and Bruttomesso [32] observed that pianists would slow the overall tempo of their playing when required to play more notes in a musical interval (beat). As will be seen below, the structure of an auditory event in terms of parameter change and event density also affects subsequent reproduction of timed actions.

\subsection{Smooth (Single-Event) and Ridged (Multiple-Event) Continuous Sounds}

The notion that the magnitude of physical energy specified by the structure an auditory event can dilate its experienced and re-enacted duration was investigated in a previously unpublished study conducted by the present authors. The task was similar to that described in Rodger and Craig [34]: participants $\left(n=38\right.$, female $=23 ; \mathbf{M}_{\text {age }}=$ 21.2 years) had to move their index finger between lateral targets to synchronise with different interval durations (1s, 2s, and 3s) specified by different sounds types (detailed below). However, in this study, a continuation paradigm was used in which 
participants heard the sounds for a set number of repetitions before having to produce timed movements at the tempo of the just-heard timing cues. Two of the sounds used were identical to those used in [34]: discrete tones and pitch ramps. A third sound type was also introduced: a series of end-to-end discrete tones rising in pitch chromatically over the duration of the interval before looping back to the initial pitch after each interval boundary. Each of these sounds is depicted as changes in auditory parameters in Fig. 2a. Pitch ramps and tone scales are designated continuous-smooth and continuous-ridged sounds, respectively. The structural difference between continuous-smooth sounds and continuous-ridged sounds is that the ridged sound's steps in pitch form perceptual sub-events within the larger looping interval-length event.

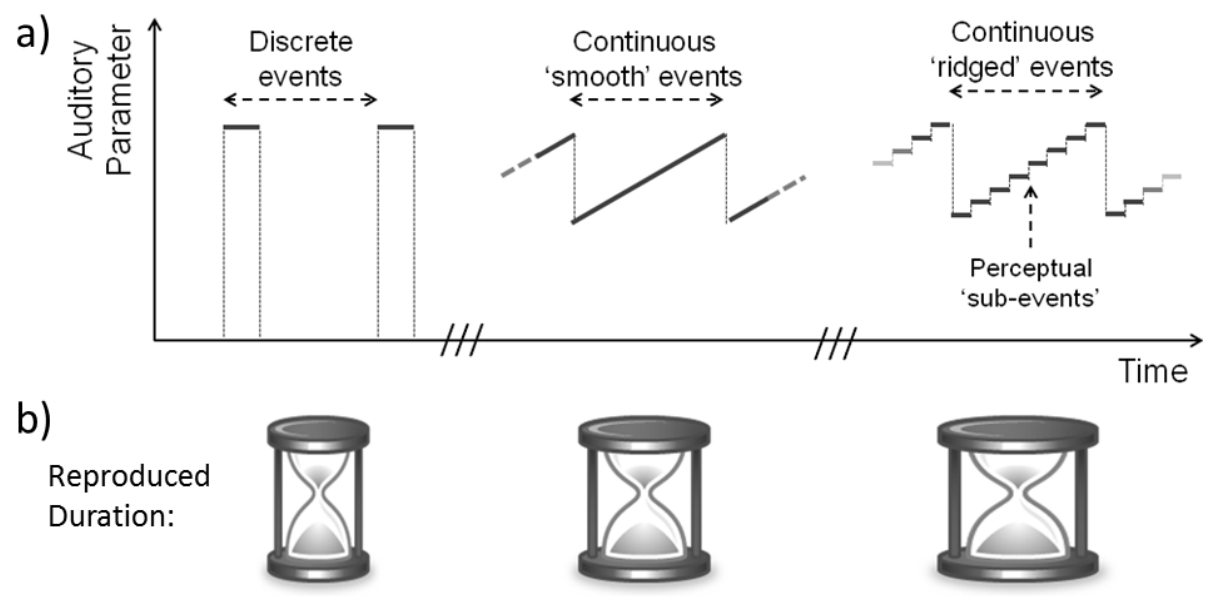

Fig. 2. Effects of auditory event structure on continuation movement timing. A): Sounds used in this study were discrete tones, continuously increasing pitch ramps (continuous-smooth), and concatenations of individual tones, which increased consecutively in pitch over the same distance as for the pitch ramps (continuous-ridged). B): Results from this study showed that participants altered the duration of their movements in a continuation task depending on the structure of the auditory event, with longest movement durations in the continuous-ridged condition, and shortest in the discrete condition.

The results from the continuation phase of this experiment are depicted symbolically in Fig. 2b, and graphically in Fig. 3. Overall, there was a significant effect of sound type on participants' reproduced interval durations $\left(F_{(2,74)}=41.96, p<\right.$ $\left..001, \mu_{p}^{2}=.53\right)$. In keeping with previous research [3, 41, 46], filled intervals were reenacted as longer in duration than empty discrete intervals. In addition, filled interval events that contained multiple sub-events (continuous-ridged sounds) were re-enacted as longer in duration than intervals that were filled with a single whole event (continuous-smooth sounds). This order of effect between sound types occurred in all duration and movement distance task conditions. The findings of this study, therefore, provide support for the proposition that the structure of auditory events (discrete/continuous; smooth/ridged) can affect the re-enaction of time in subsequent movements. Thus, the perceived temporal structure of sound echoes in movement timing, even after the sound is no longer perceptually present. 


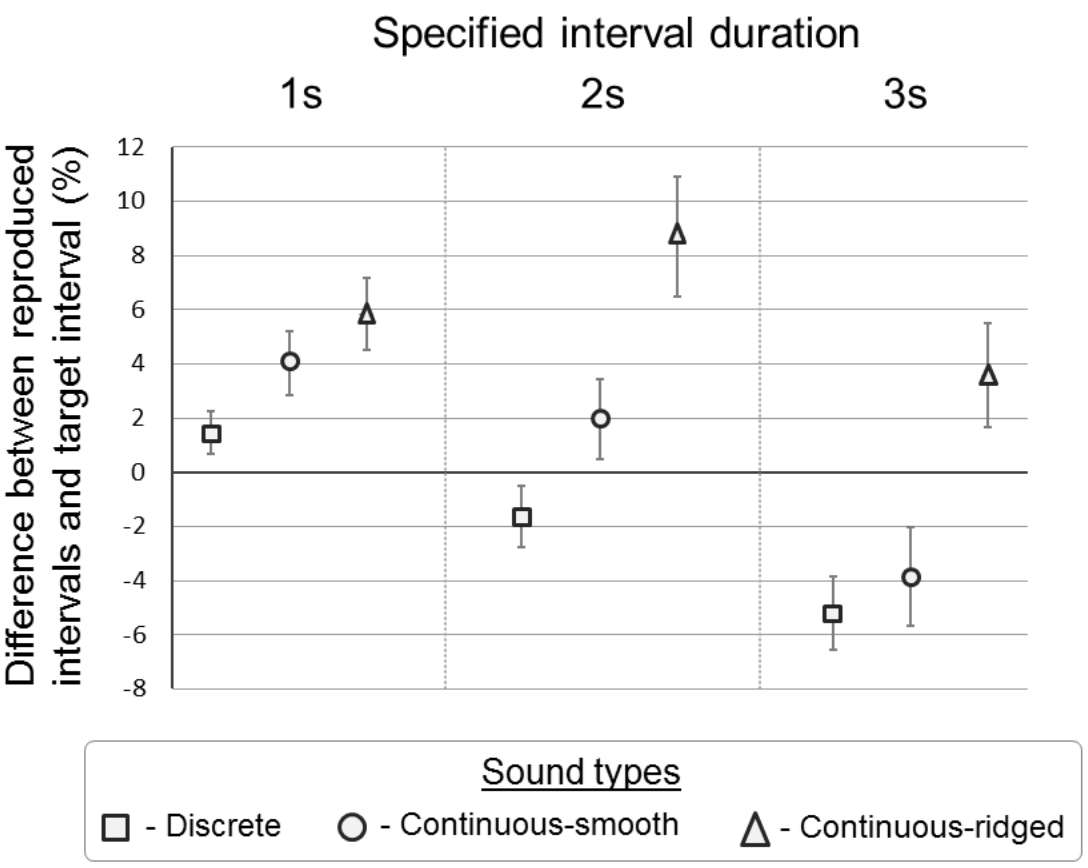

Fig. 3. Mean percentage differences between participants' reproduced interval durations in a continuation task relative to the interval durations specified by the different sound types (positive percentages indicate that reproduced intervals were longer than as specified by the previously heard sounds). Error bars denote standard error of the mean.

\subsection{Timing in Perceptual Events Mediated by Action Salience}

It should be asked at this point why the structural content of an auditory event should modulate the perceived/enacted duration of the sound. As indicated above, one explanation is that the perceived magnitude of work/energy in an event is positively related to its perceived duration [44]. The current account would take this idea further by suggesting that the reason for this has to do with the perceived action-salience of a sound: sounds that invite or suggest a greater magnitude of action effort on the part of the listener are likely to be perceived as occupying a greater expanse of time.

This proposition comes back to the ecological approach to perception and action, which states that our experience is mediated by the opportunities for action that our senses pick up. Gibson [15] said that "time is the ghost of events", intimating that the experience of time and duration are not detached from our active perception of the world, but are rather properties of the events we purposefully sift through for potential actions. In the previously described experiment, the difference in reproduced duration between the continuous-smooth and continuous-ridged sounds was that the sub-events within the ridged sounds supported the possibilities for sub-actions within the larger movements. Hence, these sounds invited a greater complexity of movement within intervals than the smooth sounds, even if this complexity was not acted upon. This idea is still very speculative, but is being further investigated by the first author. 
A more general point that should be made about timing and sound is the reciprocal nature of perception and action. Gibson said that we 'move to perceive and perceive to move' [16]. This is evidenced in research into audio-motor processes. Perceived melodic motion in a simple musical sequence affects the timing and trajectory of subsequent actions [1]. On the other side, participants are better able to detect a timing offset irregularity in a sound sequence if they first move along with a previous isochronous tone sequence than if they passively listen to the sounds [25, 40]. Hence, in trying to make sense of the perception of sound, we should also be thinking about the possible role of movement in responding to sound.

\section{Ongoing research and practical implications}

Work is currently ongoing at Queen's University Belfast to further explore the ideas laid out in this position paper. The effects of different auditory parameters on movement timing are being investigated by comparing consonant and dissonant tonal structures, as well as different continuous and discrete auditory events. Research in preparation for submission has indicated that the tonal structure of chords (consonant versus dissonant) has an effect on the temporal resonance of participants movement timing in a synchronization-continuation tasks. Timing appears to be more stable and accurate when keeping time in relation to consonant rather than dissonant tonal intervals, which may relate to the ecological preference in humans and certain animals towards consonant sounds, such as human vocalizations. Further work is currently being conducted to understand the relationships between auditory structures of sound and sensorimotor timekeeping.

In addition to these sensorimotor timing investigations, the ability of people to perform spatio-temporally constrained goal-direct actions through perceived auditory events is being studied through a number of experiments. These research projects aim to further elucidate the relationships between the structure of sounds and the structure of timed movements. Furthermore, as acknowledged in the introduction, the synthesized sounds described in this paper are not necessarily typical of those found in real-world interactions, which are much more complex in structure [4]. Research needs to be conducted to assess the extent to which the claims made here obtain when auditory events are more like those found in everyday experience.

There is also a more practical motivation for tackling these theoretical issues. A strong driving force for the current authors' engagement with these theoretical questions is the potential for using sound guides to enhance movement performance, in both motor rehabilitation and skill acquisition contexts. For example, footstep sound events have been shown to successfully convey spatio-temporal walking information to healthy participants [50], and the possibly beneficial effects of this have now been observed in a study of patients with Parkinson's disease [51]. In the latter study, patients with Parkinson's disease were found to increase their step length while listening to recordings of long strides on gravel. Interestingly, the advantage of these sounds was also observed when patients were played these sounds and then asked to walk without them, showing that the resonance of auditory events in the minds of the patients can continue to influence motor action even when such sounds have ceased. A new step has also been to synthetically sonify in real-time the swing 
phase of Parkinson's disease patients' steps, so that they can now hear the silent portion of their gait while walking [36]. This new development has shown promising benefits to patients, most likely through the augmented action-relevant feedback that these sounds provide through moving. In summary, by understanding better the ways that sounds can specify information for action, we may be able to design more optimal sensory guides that optimally scaffold motor performance for individuals with movement deficits.

\section{Conclusions}

The theoretical position outlined in this paper is that auditory sensorimotor processes are best understood by considering the structure of auditory events and the form of movement and motor timing supported by this structure. Hence, sounds in this context can be thought of as perceptual events that invite or align with particular forms of action. This can be seen in the effects of discrete and continuous sounds as distinct auditory structures on sensorimotor synchronization performance. Additionally, sound structure can influence re-enacted interval duration in a sensorimotor continuation task. Although there is much work still to be done, a case is forming for the argument that sound structure and movement timing are intimately linked when people act on sounds and that audio-motor processes are best examined by asking what is the available information for action in the structure of an auditory event. In practice, this work may have implications in the design of auditory guides as a potential strategy for movement rehabilitation.

Acknowledgments. The work presented here was partly supported by a Starting Independent Researcher's Project funded by the European Research Council (TEMPUS-G 210007 StIG) awarded to Cathy M. Craig.

\section{References}

1. Ammirante, P., Thompson, W.: Continuation Tapping to Triggered Melodies: Motor Resonance Effects of Melodic Motion. Exp. Brain. Res. 216, 51-60 (2012).

2. Bieńkiewicz, M.M.N., Rodger, M.W.M., Craig, C.M.: Timekeeping Strategies Operate Independently from Spatial and Accuracy Demands in Beat-Interception Movements. Exp. Brain. Res. 222, 241-53 (2012).

3. Buffardi, L.: Factors Affecting the Filled-Duration Illusion in the Auditory, Tactual, and Visual Modalities. Percept. Psychophys. 10, 292-294 (1971)

4. Carello, C., Wagman, J., Turvey, M.: Acoustic specification of object properties. In: Anderson, J.D. and Anderson, B.F. (eds.) Moving Image Theory: Ecological considerations. pp. 79-104. Southern Illinois University Press (2005).

5. Cisek, P., Kalaska, J.F.: Neural Mechanisms for Interacting with a World Full of Action Choices. Annu. Rev. Neuroscience. 33, 269-98 (2010).

6. Clark, A.: Being There: Putting Brain, Body, and World Together Again. MIT Press, Cambridge, MA (1997).

7. Craig, C.M., Delay, D., Grealy, M.A., Lee, D.N.: Guiding the Swing in Golf Putting. Nat. 405, 295-296 (2000). 
8. Craig, C.M., Lee, D.N.: Neonatal Control of Sucking Pressures: Evidence for an Intrinsic (tau)-Guide. Exp. Brain. Res. 124, 371-382 (1999).

9. Craig, C.M., Pepping, G.J., Grealy, M.A.: Intercepting Beats in Pre-Designated Target Zones. Exp. Brain. Res. 165, 490-504 (2005).

10. Cutting, J.: Six Tenets for Event Perception. Cogn. 10, 71-78 (1981).

11. Delignières, D., Torre, K.: Event-Based and Emergent Timing: Dichotomy or Continuum? A Reply to Repp and Steinman (2010). J. Motor. Behav. 43, (2011).

12. Dewey, J.: The Reflex Arc Concept in Psychology. Psychol. Rev. 3, (1896).

13. Elliott, M.T., Welchman, a E., Wing, a M.: Being Discrete Helps Keep to the Beat. Exp. Brain. Res. 192, 731-7 (2009).

14.Gaver, W.: What in the World Do We Hear?: An Ecological Approach to Auditory Event Perception. Ecol. Psychol. 5, 1-29 (1993).

15. Gibson, J.J.: Events Are Perceived but Time is Not. In: Fraser, J.T. and Lawrence, N. (eds.) The Study of Time, Vol. 2. pp. 295-301. Springer, New York (1975).

16. Gibson, J.J.: The Ecological Approach to Visual Perception. Houghton Mifflin, Boston, MA (1979).

17. Grassi, M., Pavan, A.: The Subjective Duration of Audiovisual Looming and Receding Stimuli. Atten. Percept. Psychophys. 74, 1321-33 (2012).

18. Hurley, S.L.: Consciousness in Action. Harvard University Press, Cambridge, MA (1998).

19. Lee, D.N.: A Theory of Visual Control of Braking Based on Information About Time to Collision. Percept. 5, 437-459 (1976).

20. Lee, D.N.: Guiding Movement by Coupling Taus. Ecol. Psychol. 10, 221-250 (1998).

21. Lee, D.N.: General Tau Theory: Evolution to Date. Special Issue: Landmarks in Perception. Percept. 38, 837-858 (2009).

22. Lee, D.N., Georgopoulos, A.P., Clark, M.J., Craig, C.M., Port, N.L..: Guiding Contact by Coupling the Taus of Gaps. Exp. Brain. Res. 139, 151-159 (2001).

23. Lee, D.N., Lishman, J.R., Thomson, J.A.: Regulation of Gait in Long Jumping. J. Exp. Psychol. Hum. Percept. Perform. 8, 448-459 (1982).

24. Lemoine, L., Delignières, D.: Detrended Windowed (Lag One) Autocorrelation: A New Method for Distinguishing Between Event-Based and Emergent Timing. Q. J. Exp. Psychol. 62, 585-604 (2009).

25. Manning, F., Schutz, M.: "Moving to the beat” Improves Timing Perception. Psychon. Bull. Rev. 20, 1133-1139. (2013).

26. Neuhoff, J.G.: An Adaptive Bias in the Perception of Looming Auditory Motion. Ecol. Psychol. 13, 87-110 (2001).

27. Noulhaine, M., Mella, N., Samson, S., Ragot, R., Pouthas, V.: How Emotional Auditory Stimuli Moderate Time Perception. Emot. 7, 697-704 (2007).

28. Phillips, D.P., Hall, S.E., Boehnke, S.E.: Central Auditory Onset Responses, and Temporal Asymmetries in Auditory Perception. Hear. Res. 167, 192-205 (2002).

29. Repp, B.H.: Phase Correction, Phase Resetting, and Phase Shifts After Subliminal Timing Perturbations in Sensorimotor Synchronization. J. Exp. Psychol. Hum. Percept. Perform. 27, 600-621 (2001).

30. Repp, B.H.: Rate Limits in Sensorimotor Synchronization With Auditory and Visual Sequences: The Synchronization Threshold and the Benefits and Costs of Interval Subdivision. J. Mot. Behav. 35, 355-370 (2003).

31. Repp, B.H.: Sensorimotor Synchronization: A Review of the Tapping Literature. Psychon. Bull. Rev. 12, 969-992 (2005).

32. Repp, B., Bruttomesso, M.: A Filled Duration Illusion in Music: Effects of Metrical Subdivision on the Perception and Production of Beat Tempo. Adv. Cogn. Psychol. 5, 114 134 (2009).

33. Repp, B., Steinman, S.: Simultaneous Event-Based and Emergent Timing: Synchronization, Continuation, and Phase Correction. J. Motor. Behavior. 42, 111-26 (2010). 
34. Rodger, M.W.M., Craig, C.M.: Timing Movements to Interval Durations Specified by Discrete or Continuous Sounds. Exp. Brain. Res. 214, 393-402 (2011).

35. Rodger, M.W.M., O’Modhrain, S., Craig, C.M.: Temporal Guidance of Musicians' Performance Movement is an Acquired Skill. Exp. Brain. Res. 226, 221-230 (2013).

36. Rodger, M.W.M., Young, W.R., Craig, C.M.: Synthesis of Walking Sounds for Alleviating Gait Disturbances in Parkinson's Disease. IEEE T. Neural. Syst. Rehabil. Eng. 22, 543-548 (2014).

37. Schogler, B., Pepping, G-J., Lee, D.N. TauG-Guidance of Transients in Expressive Musical Performance. Exp. Brain. Res. 189, 361-372 (2008).

38. Shaw, R.E., Flascher, O.M., Mace, W.M.: Dimensions of Event Perception. In: Prinz, W. and Bridgeman, B. (eds.) Handbook of Perception and Action: Volume 1. pp. 345-395. Academic Press Ltd, London (1996).

39. Studenka, B.E., Zelaznik, H.N.: Circle Drawing Does Not Exhibit Auditory-Motor Synchronization. J. Motor. Behav. 43, 185-91 (2011).

40. Su, Y-H., Poppel, E.: Body Movement Enhances the Extraction of Temporal Structures in Auditory Sequences. Psychol. Res. 76, 373-382 (2012).

41. Thomas, E., Brown, I.: Time Perception and the Filled-Duration Illusion. Percept. Psychophys. 16, 449-458 (1974).

42. Turvey, M.T., Shaw, R.E., Reed, E.S., Mace, W.M.: Ecological Laws of Perceiving and Acting: In reply to Fodor and Pylyshyn. Cogn. 9, 237-304 (1981).

43. Varlet, M., Marin, L., Issartel, J., Schmidt, R.C., Bardy, B.G.: Continuity of visual and auditory rhythms influences sensorimotor coordination. PloS One. 7, e44082 (2012).

44.Walsh, V.: A Theory of Magnitude: Common Cortical Metrics of Time, Space and Quantity. Trends. Cogn. Sci. 7, 483-488 (2003).

45. Wearden, J.H.: Do Humans Possess an Internal Clock with Scalar Timing Properties? Learn. Motiv. 22, 59-83 (1991).

46. Wearden, J.H., Norton, R., Martin, S., Montford-Bebb, O.: Internal Clock Processes and the Filled-Duration Illusion. J. Exp. Psychol. Hum. Percept. Perform. 33, 716-729 (2007).

47. Wing, A., Kristofferson, A.: Response Delays and the Timing of Discrete Motor Responses. Percept. Psychophysic. 14, 5-12. (1973).

48. Wing, A.M., Doumas, M., Welchman, A.E.: (2010). Combining Multisensory Temporal Information for Movement Synchronisation. Exp. Brain. Res. 200, 277-282 (2010).

49. Withagen, R., de Poel, H.J., Araujo, D. Pepping, G.J.: Affordances Can Invite Behavior: Reconsidering the Relationship Between Affordances and Agency. New. Ideas. Psychol. 30, 250-258 (2012).

50. Young, W., Rodger, M., Craig, C.M.: Perceiving and Reenacting Spatiotemporal Characteristics of Walking Sounds. J. Exp. Psychol. Hum. Percept. Perform. 39, 464-76 (2013).

51. Young, W.R., Rodger, M.W.M., Craig, C.M.: Auditory Observation of Stepping Actions Can Cue Both Spatial and Temporal Components of Gait in Parkinson's Disease Patients. Neuropsychologia. 57, 140-153 (2014). 